\section{Análise dos efeitos da utilização da tala seriada em crianças portadoras de paralisia cerebral: uma revisão sistemática da literatura}

\section{Analysis of the effects of the use of serial casting in children with cerebral palsy: a systematic review of the literature}

\author{
Natália Coutinho Calcagno 1 \\ Tatiana Pessoa da Silva Pinto 2 \\ Daniela Virgínia Vaz 3 \\ Marisa Cotta Mancini 4 \\ Rosana Ferreira Sampaio 5
}

\begin{abstract}
Objectives: to perform a systematic literature review through a careful survey of scientific articles investigating serial casting effects in children clinically diagnosed with spastic cerebral palsy.

Methods: systematic literature survey performed during the period of March to August 2004, in Medline, Lilacs and PEDro electronic databases published in English and Portuguese from 1980 to 2004. The evidence level of the articles studied was rated according to the Magee scale.

Results: the four articles included in this review met all the inclusion criteria and were rated poor according to the Magee scale (agreement index Kappa $K=1.0$ ) due to the methodological limitations determined.

Conclusions: the evidence used in this systematic review was insufficient to support the use of serial casting in children with cerebral palsy. Further research is required to fulfill the need of higher methodological standards to validate evidence based practice.
\end{abstract}

Key words Cerebral palsy, Physcal therapy techniques, Child

\section{Resumo}

Objetivos: realizar uma revisão sistemática da literatura através da seleção e análise criteriosa de artigos científicos que investigaram os efeitos da tala seriada em crianças com diagnóstico clínico de paralisia cerebral espástica.

Métodos: busca sistemática na literatura foi realizada no período de março a agosto de 2004, nas bases de dados eletrônicas Medline, Lilacs e PEDro publicados nas línguas inglês e português, no período de 1980 a 2004. O nível de evidência dos artigos estudados foi qualificado utilizando-se a escala Magee.

Resultados: os quatro artigos incluídos nessa revisão preencheram todos os critérios de inclusão e foram classificados como fracos de acordo com a escala Magee (índice de concordância Kappa $K=1,0$ ) devido às limitações metodológicas apresentadas.

Conclusões: as evidências utilizadas nessa revisão sistemática não apresentam nível que possa suportar a utilização clínica da tala seriada em crianças com paralisia cerebral. Futuras pesquisas devem ser realizadas em observância à necessidade de maior rigor metodológico dos estudos para a fundamentação da prática baseada em evidência.

Palavras-chave Paralisia cerebral, Técnicas de fisioterapia, Criança 


\section{Introdução}

Paralisia cerebral (PC) é definida como condição permanente não progressiva resultante de lesão do cérebro em desenvolvimento, levando à desordem de movimento e postura. ${ }^{1}$ Esta condição é bastante freqüente entre as patologias infantis, ${ }^{2}$ tendo incidência na população de aproximadamente dois em cada 1000 nascidos vivos, podendo chegar a sete por 1000 em países em desenvolvimento. ${ }^{3}$ As alterações características dessa condição que contribuem para a disfunção motora apresentada por pacientes com PC incluem problemas neuromusculares e musculoesqueléticos, tais como espasticidade, distonia, contraturas musculares, deformidades ósseas, incoordenação, perda de controle motor seletivo e fraqueza muscular. 4

As modificações nos sistemas neuro-musculoesqueléticos de crianças com PC favorecem padrões de marcha que se caracterizam por déficit na geração de força muscular, instabilidade e aumento do gasto energético em relação a crianças típicas. 5 Muitos dos padrões de marcha exibidos pelas crianças com PC envolvem movimentação anormal de tornozelo, sendo muito comum a presença do componente eqüino. 6 Essa compensação secundária é definida como uma limitação de dorsoflexão passiva em amplitude além da posição neutra do tornozelo, 7 estando associada a um apoio digitígrado durante a fase de choque, e também a uma redução do tempo da fase de apoio e à flexão plantar do tornozelo na fase de balanço. 6

Nesse contexto, o tratamento disponibilizado por profissionais da reabilitação objetiva prevenir a deformidade em eqüino, obter e manter o apoio plantígrado do pé e melhorar o padrão de marcha. Em crianças com idades inferiores à seis anos, uma abordagem conservadora é geralmente aconselhada, tanto pelo seu índice de sucesso (menor probabilidade de eqüino recorrente) quanto para evitar os riscos potenciais associados à cirurgia de alongamento do tendão do tríceps sural (anestesia, infecção, alongamento excessivo e cicatriz hipertrófica). ${ }^{8}$ Algumas das intervenções não-cirúrgicas comumente utilizadas para a melhora da marcha dessa população incluem injeção intramuscular de álcool ou toxina botulínica A e procedimentos de fisioterapia tais como o uso de órtese tornozelo-pé e a tala seriada.7,9

A tala seriada pode ser definida como um processo de aplicação e remoção sucessiva de talas corretivas para aumento da extensibilidade dos tecidos moles que circundam a articulação tratada, resultando em ganho de amplitude de movimento $(\mathrm{ADM})$ por manter um alongamento passivo prolon- gado em uma amplitude submáxima. $10 \mathrm{O}$ procedimento geral de aplicação da tala seriada envolve extensão manual da articulação fixada em flexão mantendo seu posicionamento em neutro e cobertura da região com uma camada de malha tubular e uma de espuma circular. ${ }^{11}$ A posição alongada é mantida por seis camadas de tala rígida recobrindo a camada de espuma. Cada tala é modificada periodicamente de acordo com o protocolo, sendo mais comumente utilizado o intervalo de cinco a sete dias. A cada mudança de tala aplica-se um alongamento adicional na musculatura, e a nova tala é moldada na angulação atingida. O tratamento é interrompido quando é atingida a extensão máxima possível ou quando após duas mudanças na tala não houver redução na limitação da amplitude de movimento passivo de extensão. 10

São vários os efeitos da tala seriada descritos na literatura, dentre eles aumento da ADM articular,5,7,9,12 redução da resistência articular ao movimento, 12,13 aumento do comprimento muscular,12,13 diminuição da excitabilidade reflexa, 5,13 deslocamento do ponto ótimo de geração de força para amplitudes mais alongadas, 5,13 melhora do padrão eqüino na marcha5,7,9 e ganho de função motora grossa, 14 além de um resultado positivo em relação a satisfação dos pais quanto ao tratamento. ${ }^{7}$ Entretanto, a comprovação científica sobre esses efeitos encontra-se ainda pouco sistematizada, dificultando as indicações desta intervenção num contexto de prática baseada em evidência. A utilização de evidências científicas no processo de tomada de decisão clínica está se tornando uma prática cada vez mais importante e presente entre os profissionais da saúde. 15 Nesse sentido, uma revisão sistemática da literatura auxilia o profissional da reabilitação, disponibilizando uma síntese das evidências disponíveis na literatura sobre determinado procedimento terapêutico, com rigor metodológico. 16

O objetivo desse estudo foi realizar uma revisão sistemática da literatura através da seleção e análise criteriosa de artigos científicos que investigaram os efeitos da tala seriada em crianças com diagnóstico clínico de PC espástica.

\section{Métodos}

Uma busca sistemática na literatura foi realizada no período de março a agosto de 2004, nas bases de dados eletrônicas Medline (National Library of Medicine), Lilacs (Literatura Latino-americana e do Caribe em Ciências da Saúde), e PEDro (Physiotherapy Evidence Database). Esta busca priorizou 
Resultado da apreciação crítica da qualidade da evidência dos artigos segundo a escala Magee.

\begin{tabular}{|c|c|c|c|c|}
\hline & $\begin{array}{c}\text { Brouwer } \\
\text { et al.13 (1998) }\end{array}$ & $\begin{array}{c}\text { Corry } \\
\text { et al.9(1998) }\end{array}$ & $\begin{array}{c}\text { Brouwer } \\
\text { et al.5 (2000) }\end{array}$ & $\begin{array}{l}\text { Cottalorda } \\
\text { et al.7 (2000) }\end{array}$ \\
\hline Tipo de estudo & M & A & M & B \\
\hline Controle de fatores de confusão & B & B & $\mathrm{B}$ & M \\
\hline Consentimento dos participantes do estudo & A & B & A & B \\
\hline Descrição e adequação do tratamento fisioterápico & A & B & A & M \\
\hline \multicolumn{5}{|l|}{ Método de coleta dos dados } \\
\hline Características neuromusculo-esqueléticas & B & B & B & B \\
\hline Características funcionais & N/A & B & B & N/A \\
\hline \multicolumn{5}{|l|}{ Proporção de sujeitos começando e terminando o estudo } \\
\hline Antes & A & A & A & B \\
\hline Após & A & A & A & B \\
\hline Follow-up & A & A & A & B \\
\hline Validade externa & $\mathrm{S}$ & $\mathrm{S}$ & $\mathrm{s}$ & $\mathrm{S}$ \\
\hline Análise estatística & $S$ & $\mathrm{~S}$ & $\mathrm{~s}$ & $\mathrm{~N}$ \\
\hline Classificação final & Fraco & Fraco & Fraco & Fraco \\
\hline
\end{tabular}

$\mathrm{A}=$ alta adequação; $\mathrm{M}=$ moderada adequação; $\mathrm{B}=$ baixa adequação, $\mathrm{S}=\operatorname{sim}, \mathrm{N}=$ não.

estudos publicados sobre a aplicação da tala seriada no tratamento de crianças com PC espástica. As palavras-chave utilizadas foram: casting, repeat casts e stretching casts em combinação com cerebral palsy e com spasticity. A busca foi limitada para os artigos publicados no período de janeiro de 1980 a agosto de 2004.

Estes critérios de inclusão foram previamente estabelecidos com o objetivo de definir claramente a adequação da literatura encontrada para esse estudo de revisão. Os critérios de inclusão definidos para a seleção dos artigos foram: a) intervenção definida como tala seriada; b) intervenção da tala seriada definida como variável independente ou primária; c) pesquisa realizada com seres humanos; d) artigos publicados em inglês ou português; e) os participantes deveriam ter diagnóstico de paralisia cerebral e apresentar espasticidade; f) os participantes deveriam ser crianças (até a idade de 16 anos). Os critérios de exclusão foram desenvolvidos para limitar a influência que possíveis fatores externos pudessem ter nos efeitos da tala seriada. Os critérios de exclusão para esta revisão foram: a) intervenção cirúrgica; b) intervenção utilizando tala seriada associada a outro procedimento como, por exemplo, a aplicação de toxina botulínica ou outro medicamento; c) intervenção com tala definida como tala de redução de tônus (inibitiva) ou órtese tornozelopé; d) estudos de caso ou com a amostra menor que cinco crianças.

\section{Avaliação da qualidade dos artigos revisados}

Os artigos encontrados nas buscas foram submetidos a uma avaliação da qualidade da evidência por dois examinadores independentes, através de uma adaptação da escala de Magee. 16 Essa escala tem sido utilizada em pesquisas na área de reabilitação16 e proporciona uma avaliação de ítens relevantes à prática fisioterápica. A escala Magee consiste em três fases: uma etapa inicial de avaliação preliminar da relevância do estudo para a revisão sistemática, de acordo com os critérios de inclusão e exclusão pré-determinados, sendo selecionados para a próxima etapa os artigos que obedecerem a esses critérios. Na segunda etapa é realizada a apreciação crítica dos estudos, constituída por nove itens (listados na Tabela 1) que devem ser julgados como: alta adequação (A), moderada adequação (M) ou baixa adequação (B), ou quanto à presença (S) ou ausência da informação (N).

Nos casos de discordância entre avaliadores na pontuação de cada item, a razão de sua ocorrência era registrada como descuido do examinador ou diferenças na interpretação do estudo. Para a classificação final de cada item as divergências eram discutidas até que um consenso entre os autores fosse atingido. A avaliação quantitativa da concordância entre os dois examinadores foi realizada calculando-se o índice Kappa ponderado para três categorias (A, M e B). A classificação final dos 
trabalhos foi realizada em uma última etapa considerando uma avaliação global. Na avaliação final, todos os itens, com exceção de validade externa e utilização de teste estatístico, têm o mesmo peso na avaliação da qualidade da evidência. Cada avaliador classificou os trabalhos como fraco, moderado ou forte, dependendo de sua pontuação nos critérios da apreciação crítica.

\section{Extração dos dados dos artigos}

Após seleção criteriosa dos artigos dessa revisão, foram extraídas e resumidas de forma padronizada as informações disponíveis nos mesmos, que incluem: autor(es), diagnóstico clínico, participantes (número, divisão dos grupos do estudo), faixa etária dos participantes, topografia, gravidade do comprometimento motor baseado na escala Gross Motor Function Classification System (GMFCS), desfechos avaliados, detalhes do programa de tratamento (tempo total de tratamento, frequiência de troca das talas), análise estatística utilizada para avaliação dos efeitos, e resultados encontrados.

O GMFCS é uma escala ordinal de cinco níveis, que tem sido amplamente utilizada na classificação do comprometimento motor de crianças portadoras de PC. 17 Os níveis do GMFCS variam de acordo com as limitações funcionais apresentadas pela criança e com a necessidade de equipamentos para locomoção, sendo classificada no nível I quando a criança apresenta deambulação independente sem restrição em ambientes externos e no nível V quando a criança apresenta mobilidade gravemente limitada mesmo com o uso de tecnologia assistiva.

\section{Resultados}

A pesquisa inicial realizada nas bases de dados eletrônicas identificou 97 referências, sendo 45 artigos resultantes da combinação das palavraschave casting e cerebral palsy; 40 artigos da combinação dos termos casting e spasticity; um artigo do cruzamento repeat casts e cerebral palsy; um artigo da combinação repeat casts e spasticity; três artigos de stretching casts em combinação com spasticity e sete artigos do cruzamento stretching casts e cerebral palsy. Cruzadas todas as buscas, foram identificados 26 artigos repetidos; retirando-se as duplicatas, totalizaram 71 trabalhos. Desses, 19 artigos foram pré-selecionados pelo conteúdo do título de acordo com os critérios de inclusão e exclusão estabelecidos. Após a leitura dos resumos, foi feita nova seleção com base nos mesmos critérios, sendo que
11 resumos foram excluídos por serem estudos de caso ou revisão de literatura, por envolverem intervenção cirúrgica ou aplicação de toxina botulínica anteriormente ou em associação à aplicação da tala, pelo fato de os sujeitos serem adultos ou indivíduos com idade superior a 16 anos, ou a intervenção consistir em tala de redução de tônus e não tala seriada. Dessa forma, oito artigos foram selecionados para avaliação. Após leitura dos artigos, um estudo foi excluído pois a intervenção primária não consistia em tala seriada, mas órtese tornozelo-pé (short leg casting), o segundo foi excluído por associar terapia anti-espástica ao uso da tala, o terceiro por incluir no protocolo de intervenção o uso de tala noturna e outras intervenções após o término do período de aplicação de talas seriadas e o quarto por não ser preciso em relação ao diagnóstico clínico dos participantes. Foram incluídos para a etapa de apreciação crítica os quatro artigos resultantes. As informações extraídas de cada trabalho estão apresentadas de forma resumida na Tabela 2 .

A concordância entre os autores que realizaram a apreciação crítica dos artigos foi elevada, com índice Kappa ponderado $K=0,90$. As discordâncias encontradas nas avaliações de cada item foram discutidas entre os autores até que se atingisse um consenso. Todos os quatro estudos incluídos nesta revisão sistemática foram considerados fracos na classificação global, por apresentarem pelo menos um ítem pontuado como baixa adequação na apreciação crítica. A Tabela 1 apresenta informações detalhadas da avaliação da qualidade de evidência dos artigos.

\section{Fatores que contribuíram para classificação final dos artigos}

Vários fatores devem ser considerados na análise da qualidade metodológica de artigos incluídos em uma revisão sistemática. Os seguintes fatores contribuíram diretamente para a classificação final dos artigos como fracos: desenho metodológico do estudo; controle de fatores de confusão como idade, sexo, patologias ou lesões associadas, comprometimento topográfico e gravidade da paralisia cerebral, e procedimentos terapêuticos prévios; consentimento para participação; descrição do tratamento fisioterápico; métodos de coleta de dados e porcentagem de sujeitos que iniciaram e terminaram o estudo (Tabela 1).

Quanto ao desenho metodológico apenas dois artigos eram estudos do tipo randomizado, sendo o restante constituído de desenhos quase-experimentais, o que contribuiu para o comprometimento da 
Resumo comparativo dos artigos que preencheram os critérios de relevância.

\begin{tabular}{|c|c|c|c|c|}
\hline Autor & $\begin{array}{l}\text { Brouwer et al. } \\
\text { (1998) }\end{array}$ & $\begin{array}{l}\text { Corry et al. } \\
\text { (1998) }\end{array}$ & $\begin{array}{l}\text { Brouwer et al. } \\
(2000)\end{array}$ & $\begin{array}{l}\text { Cottalorda et al. } \\
(2000)\end{array}$ \\
\hline Diagnóstico clínico & PC espástica & PC espástica & PC espástica ou Eqüino Idiopático & PC espástica \\
\hline Participantes & $\begin{array}{l}20 \text { crianças ( } 10 \text { PC } \\
\text { espástica e } 10 \text { típicas) }\end{array}$ & $\begin{array}{l}20 \text { crianças } \mathrm{PC}(10 \text { grupo tala } \\
\text { e } 10 \text { grupo TBA) }\end{array}$ & $\begin{array}{l}16 \text { crianças ( } 8 \text { PC e } 8 \text { com } \\
\text { eqüino idiopático) }\end{array}$ & 20 crianças (30 tornozelos) \\
\hline Idade & 4-14 anos & $2-9$ anos & $3-12$ anos & $2-6$ anos \\
\hline Topografia & Hemiplegia (4) e diplegia (6) & $\begin{array}{l}\text { Hemiplegia (8), diplegia (11) } \\
\text { e quadriplegia (1) }\end{array}$ & Hemiplegia e diplegia & Hemiplegia (10) e diplegia (10) \\
\hline Gravidade & Não descrito & Nível I/II do GMFCS & Nível I/II do GMFCS & Nível I/II do GMFCS \\
\hline Detalhes do programa & $\begin{array}{l}\text { Tala seriada por três semanas } \\
\text { (troca semanal) }\end{array}$ & $\begin{array}{l}\text { Tempo total de tratamento: } 12 \\
\text { semanas. Não descreve protocolo } \\
\text { da tala seriada }\end{array}$ & $\begin{array}{l}\text { Tala seriada por três a seis semanas } \\
\text { (troca em } 1 \text { a } 2 \text { sema-nas) }\end{array}$ & $\begin{array}{l}\text { Tempo total de tratamento: } \\
\text { três semanas; aplicadas três } \\
\text { talas sucessivas. Após esse } \\
\text { período, sessões de fisioterapia } \\
\text { e uso de órtese tornozelo-pé. }\end{array}$ \\
\hline Desfechos avaliados & $\begin{array}{l}\text { 1) Excitabilidade reflexa } \\
\text { 2) Força isométrica FP } \\
\text { 3) ADM passiva de DF }\end{array}$ & $\begin{array}{l}\text { 1) ADM passiva de DF } \\
\text { (joelho em flexão e extensão) } \\
\text { 2) Tônus da panturrilha } \\
\text { 3) Parâmetros da marcha }\end{array}$ & $\begin{array}{l}\text { 1) Excitabilidade reflexa } \\
\text { 2) Força isométrica FP } \\
\text { 3) Extensibilidade FP } \\
\text { 4) Parâmetros da marcha }\end{array}$ & $\begin{array}{l}\text { 1) ADM passiva DF } \\
\text { (joelho em flexão e extensão) } \\
\text { 2) Satisfação dos pais (somente } \\
\text { ao final de todo o }\end{array}$ \\
\hline
\end{tabular}


Resumo comparativo dos artigos que preencheram os critérios de relevância.

\begin{tabular}{|c|c|c|c|c|}
\hline Autor & $\begin{array}{l}\text { Brouwer et al. } \\
\text { (1998) }\end{array}$ & $\begin{array}{l}\text { Corry et al. } \\
\text { (1998) }\end{array}$ & $\begin{array}{l}\text { Brouwer et al. } \\
(2000)\end{array}$ & $\begin{array}{l}\text { Cottalorda et al. } \\
(2000)\end{array}$ \\
\hline Análise estatística & $\begin{array}{l}\text { Teste } t, \text { ANOVA, comparações } \\
\text { post-hoc }(\alpha=0.05)\end{array}$ & $\begin{array}{l}\text { Wilcoxon, Mann-Whitney U, } \\
\text { teste Fisher }(\alpha=0.05)\end{array}$ & Teste $\mathrm{t}$, ANOVA, $(=0.05)$ & Não descreve \\
\hline Efeitos encontrados & $\begin{array}{l}\text { 1) Excitabilidade reflexa: Pré: } \\
\text { RE precoce na DF }(p<0,0001) \text { e } \\
\downarrow \text { PF de FP }(p<0,0001) \text { em PCs. } \\
\text { Pós: } \uparrow \text { limiar do RE }(p<0,03) \text {. } \\
\text { 2) Força isométrica FP: pré }=\text { pós } \\
(p>0,32) \\
\text { 3) } \uparrow \text { ADM passiva de DF }(p<0.01)\end{array}$ & $\begin{array}{l}\text { 1) ADM: duas semanas: } \uparrow \text { ADM nos } \\
\text { dois grupos, } 12 \text { semanas: sem } \\
\text { alteração nos dois grupos) } \\
\text { 2) Tônus: duas semanas: alterações } \\
\text { quase significativas no grupo tala } \\
\text { ( } p=0,065) .12 \text { semanas: sem } \\
\text { alterações significativas no grupo } \\
\text { tala. } \downarrow \text { efeito grupo tala >grupo } \\
\text { TBA ( } p=0,03 \text { ). } \\
\text { 3) Marcha: melhoras padrão marcha } \\
\text { nos dois grupos. } 12 \text { semanas: } \\
\text { Łefeito nos dois grupos }\end{array}$ & $\begin{array}{l}\text { 1) Excitabilidade reflexa: Pré: } \\
\text { <limiar. RE em PCs }(p<0,001) \text {. } \\
\text { Pós: } \downarrow \text { excitabilidade do } \\
\text { reflexo em PCs }(p<0,05) \\
\text { 2) Força isométrica FP: } \\
\text { PC <idiopático no pré }(p<0,05) \\
\text { e ao longo do estudo }(p<0,001) \quad 2) \\
\text { e não mudou após intervenção } \\
\text { ( } p>0,05) \text {. } \\
\text { 3) Extensibilidade FP: } \uparrow \text { ADM e } \downarrow \\
\text { resistência ao alongamento nos } \\
\text { dois grupos ( } p<0,01) \\
\text { 4) Parâmetros da marcha: } \\
\text { velocidade e comprimento do } \\
\text { passo sem mudanças nos dois grupos } \\
\text { ( } p>0,05)\end{array}$ & $\begin{array}{l}\text { 1) ADM após três semanas de } \\
\text { tala seriada: } \uparrow \text { ADM de DF } \\
\text { com joelho em flexão e ex- } \\
\text { tensão. Melhora no contato } \\
\text { inicial na marcha (plantígrado } \\
\text { ou com choque de calcanhar) } \\
\text { 2) Satisfação dos pais: Maioria } \\
\text { dos pais avaliou a melhora } \\
\text { das crianças como } \\
\text { significativa após o final da } \\
\text { intervenção }\end{array}$ \\
\hline
\end{tabular}

TBA = toxina botulínica tipo $A ; R E=$ reflexo de estiramento; $D F=$ dorsoflexão; $P F=$ pico de força; $P C=$ paralisia cerebral; FP = flexores plantares; GMFCS = Gross motor function TBA = toxina botulinica tipo $A ; R E=$ reflexo de estirament
classification system; $A D M=$ amplitude de movimento. 
qualidade da evidência da maioria deles. No item referente ao controle dos fatores de confusão, um estudo recebeu moderada adequação por falhar em dois fatores, 7 os outros três foram classificados como baixa adequação por controlarem apenas um ou dois fatores. $5,9,13$ No item referente ao consentimento para participação no estudo, dois trabalhos receberam baixa adequação por não informarem sobre a realização desse procedimento.7,9 Quanto ao item tratamento fisioterápico, um artigo recebeu baixa adequação por não descrever o procedimento da tala seriada e não ser específico para os grupos testados. ${ }^{9}$ Os quatro estudos receberam pontuação de baixa adequação no item referente ao método de coleta de dados, subitem características neuromusculoesqueléticas, devido à ausência de mensuração dos níveis de confiabilidade intraexaminadores e de descrição da validade dos instrumentos de medida. No subitem características funcionais, os dois artigos que avaliaram tal desfecho apresentaram baixa adequação. 5,9

\section{Efeitos da tala seriada nas características} neuromusculares e musculoesqueléticas

\section{Amplitude de movimento}

Todos os artigos analisados nesta revisão sistemática demonstraram efeitos positivos na avaliação pósintervenção utilizando tala seriada em relação à ADM de dorsoflexão de tornozelo. Estudos de Corry et al.,9 e Brouwer et al.,5 avaliaram esse desfecho e encontraram efeitos significativos para ganho de ADM após uso de tala seriada. O estudo de Brouwer et al. 13 também reportou aumento estatisticamente significativo dessa amplitude. O estudo de Cottalorda et al., ${ }^{7}$ apesar de relatar aumento de ADM de dorsoflexão quantificado através de goniometria, não utiliza análise estatística dos dados, sendo inconclusivo em relação a esse desfecho. Um artigo ${ }^{9}$ contrastou os grupos submetidos à intervenção com tala seriada e com toxina botulínica, e não encontrou diferenças entre os grupos em relação ao aumento da ADM pós-intervenção, que foi significativo em ambos os grupos.

Três artigos avaliaram a amplitude de dorsoflexão no período de follow-up.5,9,13 Os artigos de Brouwer et al. ${ }^{5,13}$ realizaram follow-up seis semanas após remoção da tala, porém somente o estudo de 1998 relatou resultados dessa avaliação, que indicaram aumento significativo de dorsoflexão passiva de tornozelo em relação à avaliação préintervenção. Esse aumento, porém, foi menor do que aquele evidenciado na avaliação pós-intervenção. 13 O estudo de Corry et al. ${ }^{9}$ constatou um aumento adicional na ADM de dorsoflexão no follow-up de 12 semanas no grupo submetido ao tratamento com toxina botulínica, já no grupo que utilizou tala seriada como intervenção, esse aumento não foi significativo. Segundo os autores, a diferença aparente entre os grupos submetidos à aplicação de toxina e ao procedimento da tala no momento de follow-up não foi significativa após análise estatística. ${ }^{9} \mathrm{O}$ trabalho de Cottalorda et al. ${ }^{7}$ incluiu uso de órtese noturna logo após a retirada da tala seriada, o que impede que os resultados do follow-up possam ser atribuídos exclusivamente aos efeitos da tala. Dessa forma, os resultados de follow-up apresentados por este estudo não serão discutidos nessa revisão. 7

\section{Tônus}

O tônus muscular da panturrilha foi um desfecho avaliado em dois estudos analisados nessa revisão sistemática. 9,13 Corry et al. 9 comparando um grupo submetido à tala seriada e outro à toxina botulínica verificaram uma redução significativa do tônus da panturrilha (medido através da escala de Ashworth modificada) no grupo submetido à toxina botulínica, após duas semanas; já no grupo tala seriada a diminuição não atingiu significância estatística. Após 12 semanas, houve um declínio desse efeito no grupo TBA e alterações não significativas no grupo tala seriada. O estudo de Brouwer et al.13 relatou uma diminuição significativa da resistência ao alongamento passivo de flexores plantares após o uso da tala seriada em crianças com PC. Uma redução do tônus muscular é evidenciada através deste desfecho, uma vez que a hipertonia é definida como um aumento da resistência oferecida ao alongamento passivo. ${ }^{13} \mathrm{O}$ estudo de Brouwer et al., 5 demonstra haver redução significativa da resistência ao alongamento passivo no grupo de crianças com PC e de crianças com eqüino idiopático, após o tratamento com tala seriada. 5

\section{Excitabilidade reflexa}

A excitabilidade reflexa foi investigada em dois estudos por Brouwer et al.5,13 No primeiro trabalho, a avaliação pré-intervenção evidenciou a precocidade da resposta reflexa do tríceps sural durante a dorsoflexão passiva do tornozelo em crianças com PC, se comparada a crianças típicas. Após tratamento com tala seriada, foi constatado um aumento significativo do limiar de excitabilidade, sendo que a 
amplitude em que ocorreu o reflexo de estiramento se aproximou daquela observada em crianças normais. ${ }^{13}$ Em 2000, Brouwer et al., 5 comparando os efeitos da tala seriada em crianças com paralisia cerebral e com eqüino idiopático, também verificaram que ocorreu uma redução significativa da excitabilidade reflexa em PCs, após o tratamento.

\section{Força isométrica de flexores plantares}

A força isométrica máxima gerada por flexores plantares na amplitude total de tornozelo disponível pela criança foi um desfecho que não apresentou diferença significativa após intervenção utilizando tala seriada, no trabalho de Brouwer et al.13 Outro estudo, Brouwer et al.,5 contrastando o efeito da tala seriada em crianças com PC e com eqüino dinâmico idiopático, também demonstraram não haver alteração na força isométrica máxima de flexores plantares, em amplitudes que variaram do máximo de flexão plantar até o máximo de dorsoflexão disponível, de crianças com $\mathrm{PC}$ após intervenção. $\mathrm{O}$ dois artigos de Brouwer et al.5,13 relatam um deslocamento da curva comprimento-tensão de flexores plantares para a direita, o que indica que a força isométrica máxima de tríceps sural está sendo gerada em uma amplitude de maior dorsoflexão (maior comprimento muscular) em relação ao período préintervenção.

\section{Efeitos da tala seriada nas características funcionais}

\section{Marcha}

O estudo de Corry et al. ${ }^{9}$ apresentou resultados semelhantes em relação aos parâmetros da marcha entre grupos submetidos à tala seriada e à toxina botulínica. Esses autores encontraram melhora no apoio do pé no momento do contato inicial, na pontuação total da Physician Rating Scale (PRS) modificada e melhora significativa do padrão de marcha em 2 e 12 semanas após a intervenção com tala seriada. No estudo de Brouwer et al., 5 a velocidade da marcha e o comprimento do passo foram parâmetros que não apresentaram alterações após o uso de tala seriada em crianças com PC e com eqüino dinâmico idiopático.

\section{Satisfação dos pais}

O estudo de Cottalorda et al. 7 avaliaram a satisfação dos pais quanto à utilização da tala seriada como intervenção terapêutica. Nesse estudo, 18 dos 20 pais avaliaram a melhora das crianças submetidas ao tratamento com tala seriada como sendo significativa e dois pais julgaram a magnitude da melhora como moderada.

\section{Discussão}

O uso de tala seriada no tratamento de crianças com PC tem sido reportado em recentes estudos, entretanto sua efetividade nessa população ainda não está firmemente estabelecida. 10 Várias hipóteses acerca dos mecanismos de ação da tala seriada são levantadas na tentativa de embasar a utilização desta intervenção em crianças com PC.13

$\mathrm{Na}$ literatura, existem evidências de que as alterações nas características musculares de crianças com PC podem ser conseqüentes de adaptações semelhantes às que ocorrem em modelos experimentais com animais. $18 \mathrm{Em}$ alguns estudos envolvendo animais, adaptações morfológicas que acompanham imobilização do músculo em posição encurtada incluem: diminuição do comprimento do músculo, 19 tendão 20 ou unidade músculo-tendínea; 20 redução de até $40 \%$ do número de sarcômeros em série; 19 um aumento na porcentagem de tecido conectivo no músculo;18,19 alterações na orientação do tecido conectivo intramuscular; 19 e diminuição na extensibilidade do tecido conectivo periarticular. 21 Estudos em indivíduos com PC demonstram haver um aumento da resistência do tríceps sural à movimentação passiva, em situações de ausência de atividade eletromiográfica, o que indica a presença de alterações nas propriedades musculares passivas. Além disso, são observadas também alterações na relação comprimento-tensão da musculatura dessa população. 12

Por outro lado, estudos que investigaram os efeitos da manutenção do músculo sóleo em posição alongada, por períodos maiores que uma semana, concluíram que o aumento da extensibilidade do músculo alongado resulta da adição de até $19 \%$ no número de sarcômeros em série. ${ }^{19}$ Alguns estudos têm demonstrado uma estreita associação entre o aumento do comprimento muscular e o deslocamento da curva comprimento-tensão para direita, o que tem sido interpretado como um reflexo da adição de sarcômeros. ${ }^{12,13}$

Considerando a capacidade de adaptação do tecido muscular, intervenções terapêuticas como a tala seriada são propostas com o intuito de modificar a demanda imposta sobre o tecido e ocasionar sua remodelação, objetivando otimizar a função motora 
de crianças com PC. Os trabalhos analisados nessa revisão demonstram que o uso da tala seriada no tríceps sural de crianças com PC pode provocar efeitos positivos relacionados às características neuro-músculoesqueléticas como aumento da ADM de dorsoflexão de tornozelo, 5,7,9,13 redução do tônus da panturrilha,5,9,13 aumento do limiar de excitabilidade do reflexo de estiramento.5,13 $\mathrm{O}$ deslocamento do ponto ótimo de geração de tensão (deslocamento da curva comprimento-tensão) relatado após o uso da tala seriada, 5,13 somado aos efeitos anteriores, sugere que a aplicação da tala seriada seja efetiva para causar remodelação do tecido muscular. Além dessas modificações, foram observadas também melhoras na qualidade da marcha, evidenciadas por uma diminuição do equinismo, com melhora do contato inicial do pé,5,9 sem prejuízos para a capacidade de geração de força muscular.5,13 No entanto, não foram relatados ganhos funcionais em relação à velocidade da marcha e ao comprimento do passo.

A tala seriada é também utilizada como importante intervenção para controlar contraturas e hipertonia em adultos com lesão cerebral. Uma recente revisão sistemática sobre o uso da tala seriada no tratamento da mobilidade articular e hipertonia após lesão cerebral em adultos, analisou 13 estudos que foram agrupados por área do desfecho avaliado: espasticidade, ADM e função. 15 Segundo Mortenson et al.,15 a redução da espasticidade é freqüentemente citada como um objetivo primário do uso da tala seriada, porém apenas cinco dos 13 artigos estudados pelos autores mediram propriedades da espasticidade como um desfecho. Em todos os cinco estudos foram relatados melhora em alguma extensão nos níveis de espasticidade após o tratamento com tala seriada. Entretanto, a maneira com que foi feita a definição operacional da espasticidade bem como sua mensuração variou muito entre os estudos. A ADM foi um desfecho avaliado em 10 estudos e, com exceção de um artigo, todos reportaram melhoras na ADM após o uso da tala seriada. Todavia as mensurações apresentaram falhas em oito dos estudos por não apresentarem dados de confiabilidade e validade das medidas reportadas. As tendências acerca dos efeitos da tala seriada na função motora não podem ser identificadas devido aos resultados inconsistentes encontrados pela revisão sistemática, sendo que apenas quatro dos artigos mediram aspectos de atividade motora ou deambulação. Além disso, esses quatro estudos utilizaram instrumentação diversificada para documentar o desempenho funcional, o que dificulta a comparação entre os resultados. Apesar dos efeitos benéficos do uso da tala seriada em adultos encontrados na literatura, tais como aumento da ADM, diminuição da espasticidade e melhora na função, Mortenson et al.15 concluíram que apenas os efeitos em relação à ADM tiveram evidências suficientes para suportar a prática clínica.

Os estudos analisados nessa revisão sistemática, apesar de entrarem em consenso em relação a alguns efeitos positivos do uso da tala seriada no tratamento de crianças com PC, apresentaram falhas metodológicas constatadas na apreciação crítica da escala Magee. As principais falhas dos trabalhos estão relacionadas ao tipo de desenho metodológico utilizado, à caracterização dos participantes, à descrição e especificidade do tratamento fisioterápico, e às qualidades psicométricas dos instrumentos usados na coleta de dados.

\section{Desenho metodológico dos estudos revisados}

Todos os estudos analisados nessa revisão utilizaram o desenho metodológico do tipo quase-experimental, que compara dois ou mais tratamentos não havendo um grupo controle de referência. Nesse tipo de estudo, o objetivo é determinar qual dos tratamentos é mais efetivo, não permitindo concluir se as condições de tratamento são mais efetivas que a ausência de intervenção. 22 Apesar de não ser considerado padrão ouro entre os desenhos experimentais, esse tipo de desenho metodológico tem sido bastante utilizado em pesquisas da área de reabilitação, principalmente pelas questões éticas que permeiam a não-disponibilização de intervenção para pacientes com condições de saúde crônicas e incapacitantes. 22 Nos estudos quase-experimentais, o sujeito torna-se controle dele mesmo e é avaliado em diversos momentos durante o período do estudo. Esses desenhos são menos conclusivos com relação à causalidade dos resultados encontrados, sendo possível a associação dos efeitos da intervenção com os efeitos naturais de maturação, o que pode comprometer a validade interna dos resultados. ${ }^{22}$

\section{Caracterização dos participantes dos estudos}

Nos trabalhos analisados alguns dos fatores relacionados às características da amostra variaram bastante. A faixa etária dos participantes variou entre 2 e 14 anos, sendo a maior variação observada no estudo de Brouwer et al. ${ }^{13}$ (crianças de 4 a 14 anos de idade). É sugerido na literatura a existência de diferenças na adaptação muscular em resposta ao alongamento de acordo com a idade, 23 e assim, 
amostras com variação etária muito grande poderiam interferir nos resultados apresentados.

Outro fator que apresentou grande variabilidade entre os estudos foi a topografia do comprometimento neurológico das crianças com PC. Os quatro estudos incluíram indivíduos com quadro de hemiplegia e diplegia; um estudo inclui também quadriplegia. Apesar dessa diversidade de acometimentos topográficos, os participantes de todos os estudos possuíam marcha independente, exceto no de Brouwer et al. ${ }^{13}$ que não descreveram a gravidade do comprometimento. Sendo assim, todos os participantes nos demais estudos foram classificados nos níveis I/II do GMFCS por apresentarem marcha sem restrição e não haver informações quanto a limitações em ambientes externos e na comunidade.

Embora os artigos tenham sido bastante homogêneos em relação à severidade da limitação motora das crianças, a faixa etária da amostra variou bastante em cada estudo o que dificulta a comparação dos efeitos encontrados.

\section{Tratamento fisioterápico}

A descrição do tratamento fisioterápico está bem apresentada nos trabalhos de Brouwer et al.5,13, detalhando o procedimento de aplicação da tala seriada e seus intervalos de troca, assim como a duração do tratamento fisioterápico. Os dois artigos utilizaram o mesmo procedimento de aplicação da tala seriada, que envolve o posicionamento da articulação subtalar em neutro, mantendo o alinhamento de antepé e retropé.

O intervalo de troca da tala seriada variou de uma13 a duas semanas5. O artigo de Brouwer et al.5 utiliza um intervalo variável de uma ou duas semanas para a mudança da tala e não fornecem especificação do critério de reaplicação da tala. Várias publicações envolvendo o tratamento com tala seriada em indivíduos com lesão cerebral recomendam o protocolo de troca de tala no intervalo de cinco a sete dias. ${ }^{14}$ Entretanto, não houve investigação que comprovasse que essa recomendação provocava um melhor resultado. Um estudo 10 comparou intervalos de mudanças de um a quatro dias com o convencional (cinco a sete dias) em indivíduos adultos. Os resultados desse trabalho mostraram que períodos de troca da tala seriada inferiores a cinco dias foram melhores que o convencional por promover um redução das complicações e da taxa de descontinuidade do tratamento. 10

O tempo total de tratamento diferiu entre os estudos, variando de três 13 a seis semanas. ${ }^{9}$ Nos estudos de Brouwer et al..$^{5}$ e Corry et al. ${ }^{9}$ o período de tratamento de cada criança foi fundamentado nas alterações observadas no alongamento passivo determinado pelo profissional de referência.

Nesta revisão foi verificada uma inconsistência dos resultados tanto em relação ao efeito documentado da tala seriada no tônus muscular quanto no tempo total de tratamento empregado. O estudo de Corry et al. ${ }^{9}$ descreve ausência de alteração significativa do tônus no grupo submetido à tala seriada, resultado conflitante com os resultados encontrados nos trabalhos de Brouwer et al.5,13 que relataram diminuição significativa no tônus após a intervenção. Tal discrepância pode ser devida à sensibilidade dos diferentes métodos empregados nos estudos para detectar reduções no tônus (escala de Ashworth, no primeiro estudo 9 e registro da resistência passiva registrada por um aparelho mecânico, nos demais estudos).5,13 Informações sobre a sensibilidade dos métodos não foram documentadas. É possível que a escala Ashworth, por ser uma escala ordinal e não verdadeiramente quantitativa, seja menos sensível do que a instrumentação utilizada por Brouwer et al. 5,13

\section{Métodos de coleta de dados}

Todos os artigos analisados nesta revisão falharam em relação aos métodos de coleta de dados por não relatarem níveis de confiabilidade intraexaminador, além de validade e confiabilidade da instrumentação utilizada.

Nos artigos revisados alguns dos instrumentos utilizados na avaliação dos desfechos investigados têm validade e confiabilidade descritas na literatura, apesar desses não terem sido reportadas pelos respectivos autores, como é o caso da goniometria, ${ }^{24}$ escala de Ashworth modificada 26 e Physician Rating Scale. ${ }^{27}$ As informações sobre as propriedades dos instrumentos devem ser relatadas pelos autores para maior detalhamento acerca das mensurações realizadas. Não se deve assumir que, pelo fato de somente um examinador experiente realizar todas as mensurações, a confiabilidade intraexaminador será adequada de modo a garantir a força da evidência. ${ }^{22}$ No contexto da pratica baseada em evidências, a utilidade da evidencia documentada em pesquisas depende de certa forma, da extensão na qual os profissionais clínicos podem confiar nos resultados como sendo indicadores acurados do fenômeno ou comportamento de interesse. 22

Apesar das recentes conclusões de que a tala seriada deve ser considerada como um adjunto no 
tratamento de crianças com PC,7,13 o nível de evidência dos estudos selecionados nessa revisão não suporta essa afirmação, nem consegue negá-la. Isso se deve ao fato de que os artigos analisados apresentaram limitações metodológicas que resultaram em uma baixa classificação de acordo com a escala Magee. Dessa maneira, os efeitos positivos da tala seriada no tratamento de crianças com PC encontrados pela maioria dos artigos ainda não sustentam o uso dessa intervenção na prática clínica. Estudos adicionais de maior qualidade metodológica

\section{Referências}

1. Rosen MG, Dickinson JC. The incidence of cerebral palsy. Am J Obst Gyneco 1992; 167: 417-23.

2. Campbel SK. Physical therapy for children. Philadelphia: Saunders; 1995.

3. Nottidge VA, Okogbo ME. Cerebral palsy in Ibadan, Nigeria. Dev Med Child Neurol 1991; 33: 241-5.

4. Flett PJ. Rehabilitation of spasticity and related problems in childhood cerebral palsy. J Paediatr Child Health 2003; 39: 6-14.

5. Brower B, Davidson LK, Olney SJ. Serial casting in idiopathic toe-walkers and children with spastic cerebral palsy. J Ped Orth 2000; 20: 221-5.

6. Gage JR. Gait analysis in cerebral palsy. Londres: Mac Keith; 1991.

7. Cottalorda J, Gautheron V, Metton G, Charmet E, Chavrier $\mathrm{Y}$. Toe-walking in children younger than six years with cerebral palsy. J Bone Joint Surg 2000; 82: 541-4.

8. Flett PJ, Stern LM, Waddy H, Connell TM, Seeger JD, Gibson SK. Botulinum toxin A versus fixed cast stretching for dynamic calf tightness in cerebral palsy. J Paediatr Child Health 1999; 35: 71-7.

9. Corry IS, Cosgrove AP, Duffy CM, McNeill S, Taylor TC, Graham HK. Botulinum toxin A compared with stretching casts in the treatment of spastic equinus: a randomized prospective trial. J Ped Orth 1998; 18: 304-11.

10. Pohl M, Rückriem S, Mehrholz J, Ritschel C, Strik H, Pause MR. Effectiveness of serial casting in patients with severe cerebral spasticity: a comparison study. Arch Phys Med Rehabil 2002; 83: 784-90.

11. Pohl M, Rückriem S, Strik H, Hörtinger B, Meibner D, Mehrholz J, Pause M. Treatment of pressure ulcers by serial casting in patients with severe spasticity of cerebral origin. Arch Phys Med Rehabil 2002; 83: 35-9.

12. Tardieu G, Tardieu C, Colbeau-Justin P, Lespargot A. Muscle hypoextensibility in children with cerebral palsy: II. Therapeutic implications. Arch Phys Med Rehabil 1982; 63: 103-7.

13. Brouwer B, Wheeldon RK, Stradiotto-Parker N, Allum J. Reflex excitability and isometric force production in cerebral palsy: the effect of serial casting. Dev Med Child Neurol 1998; 40: 168-75. se fazem necessários para embasar a utilização da tala seriada em crianças com PC.

\section{Conclusões}

Apesar de alguns artigos analisados nesta revisão serem consistentes em relação a determinados efeitos da tala seriada no tratamento de crianças com paralisia cerebral, o nível de evidência apresentado por eles não é suficiente para suportar o uso da tala seriada na prática clínica.

14. Phillips WE, Audet M. Use of serial casting in the management of knee joint contractures in an adolescent with cerebral palsy. Phys Ther 1990; 70: 66-8.

15. Mortenson PA, Eng JJ. The use of casts in the management of joint mobility and hypertonia following brain injury in adults: a systematic review. Phys Ther 2003; 83: 648-58.

16. McNeely ML, Torrance G, Magee DJ. A systematic review of physiotherapy for spondylolysis and spondylolisthesis. Man Ther 2003; 8: 80-91.

17. Palisano R, Rosenbaum P, Walter S, Russell D, Galuppi B. Development and reliability of a system to classify gross motor function in children with cerebral palsy. Dev Med Child Neurol 1997; 39: 214-23.

18. Williams PE, Goldspink G. Changes in sarcomere length and physiological properties in immobilized muscle. J Anat 1978; 127: 459-69.

19. Tabary JC, Tabary C, Tardieu C, Tardieu G, Goldspink G. Physiological and structural changes in the cat's soleus muscle due to immobilization at different lengths by plaster casts. J Physiol 1972; 224: 231-44.

20. Williams PE. Effect of intermittent stretch on immobilised muscle. Ann Rheum Dis 1988; 47: 1014-6.

21. Akeson WH, Woo SLY, Amiel D, Matthews JV. Biomechanical and biochemical changes in the periarticular connective tissue during contracture development in the immobilized rabbit knee. Connect Tissue Res 1974; 2: 31523.

22. Portney LG, Walkins MP. Foundations of clinical research: applications to practice. $2^{\text {nd }}$ ed. New Jersey: Prentice-Hall; 2000.

23. Gossman MR, Sahrmann SA, Rose SJ. Review of lengthassociated changes in muscle: experimental evidence and clinical implications. Phys Ther 1982; 62: 1799-808.

24. Gajdosik RL, Bohannon RW. Clinical measurement of range of motion. Review of goniometry emphasizing reliability and validity. Phys Ther 1987; 67: 1867-72.

25. Russell DJ, Avery LM, Rosenbaum PL, Raina PS, Walter $\mathrm{SD}$, Palisano RJ. Improved scaling of the gross motor function measure for children with cerebral palsy: evidence of reliability and validity. Phys Ther 2000; 80: 873-85. 
26. Blackburn M, van Vliet P, Mockett SP. Reliability of measurements obtained with the modified Ashworth scale in the lower extremities of people with stroke. Phys Ther 2002; 82: 25-34.
27. Free SM Jr, Overall JE. Validation of a physician rating scale for investigation of antianxiety effects of drugs [proceedings]. Psychopharmacol Bull 1977; 13: 8-11.

Recebido para publicação em 20 de abril de 2005

Versão final apresentada em 21 de dezembro de 2005

Aprovado em 2 de fevereiro de 2006 